Tutorial

\title{
An engineering insight into the relationship of selective cytoskeletal impairment and biomechanics of HeLa cells
}

\author{
Daniele Borin $^{\mathrm{a}, 1}$, Luca Puzzi ${ }^{\mathrm{a}, 1}$, Valentina Martinelli ${ }^{\mathrm{b}}$, Matteo Cibinel ${ }^{\mathrm{a}}$, Romano Lapasin ${ }^{\mathrm{a}}$, \\ Orfeo Sbaizero ${ }^{\text {a,* }}$
}

${ }^{\text {a }}$ Department of Engineering and Architecture, University of Trieste, Piazzale Europa 1, 34127 Trieste Italy

b International Centre for Genetic Engineering and Biotechnology, Padriciano 99, 34149 Trieste, Italy

\section{A R T I C L E I N F O}

\section{Keywords:}

AFM

HeLa

Mechanical properties

Viscoelasticity

Storage modulus

Loss modulus

\begin{abstract}
A B S T R A C T
It is widely accepted that the pathological state of cells is characterized by a modification of mechanical properties, affecting cellular shape and viscoelasticity as well as adhesion behaviour and motility. Thus, assessing these parameters could represent an interesting tool to monitor disease development and progression, but also the effects of drug treatments. Since biomechanical properties of cells are strongly related to cytoskeletal architecture, in this work we extensively studied the effects of selective impairments of actin microfilaments and microtubules on HeLa cells through force-deformation curves and stress relaxation tests with atomic force microscopy. Confocal microscopy was also used to display the effects of the used drugs on the cytoskeletal structure. In synergy with the aforementioned methods, stress relaxation data were used to assess the storage and loss moduli, as a complementary way to describe the influence of cytoskeletal components on cellular viscoelasticity. Our results indicate that F-actin and microtubules play a complementary role in the cell stiffness and viscoelasticity, and both are fundamental for the adhesion properties. Our data support also the application of biomechanics as a tool to study diseases and their treatments.
\end{abstract}

\section{Introduction}

In recent years, single-cell mechano-biology has become an emerging scientific field linking both cellular force transmission and chemomechanical pathways to physiological and pathological conditions of the cell. Mechanical forces have been identified to affect several aspects of living organisms, for example organ morphogenesis (Beloussov et al., 1994), bone formation and modelling (Duncan and Turner, 1995), differentiation and organization of skeletal muscles (Simpson et al., 1994), and central nervous system development (Van Essen, 1997). It has also been demonstrated that cells are able to face the external stresses, changing, for instance, their rates of proliferation, protein expression and extracellular matrix (ECM) production. Furthermore, due to its viscoelastic behaviour, a cell can deform, move and replicate, as well as to restore its shape after deformation. This duality has been rationalized studying the cytoskeletal components (F-actin, microtubule and intermediate filaments) held together by dynamic crosslinks that can either resist rapid deformation or allow slow morphological changes in response to constant forces. In addition, cells deformation involves complex interactions among transmembrane proteins like integrins and cytoskeletal components (Huang et al., 2004). Thus, it becomes clear that assessing the mechanical properties of cells could be used as an indicator of their biological state and could offer valuable insights into the pathogenic basis of diseases. Mechanical stresses have been demonstrated to play a key role in several pathologies, including (but not limited to) atherosclerosis ( $\mathrm{Li}$ and $\mathrm{Xu}, 2007$ ), cancer (Suresh, 2007), arthritis (Lammi, 2004) and cardiovascular diseases (Lanzicher et al., 2015a,b).

In order to evaluate the role of bio-chemo-mechanical pathways for human diseases, a systematic study of cell mechanical properties in a physiological environment is therefore mandatory. To highlight the link between mechano-transduction and pathologies, several studies have investigated the mechanical effects of loading conditions on cells using either commercial devices such as the Flexercell system (Vande Geest et al., 2004) or custom built devices to apply strains in two-dimensional (2D) (Gorfien et al., 1989; Kulik and Alvarado, 1993; Sarraf et al., 2002) or three-dimensional (3D) cell cultures (Isenberg and Tranquillo, 2003; Kim et al., 1999; Seliktar et al., 2001). Among all the techniques, atomic force microscopy (AFM) (Binnig et al., 1986) showed a great potential in the assessment of cell mechanics due to its nanometric

\footnotetext{
* Corresponding author at: Department of Engineering and Architecture, University of Trieste, Via Valerio 10, 34127 Trieste, Italy.

E-mail address: sbaizero@units.it (O. Sbaizero).

1 Co-first authorship.
} 
resolution, providing both excellent morphological evidence and the possibility to perform measurements in liquid, thus allowing to analyse cells in their physiological environment.

Defects in the cytoskeleton structure represent the effects or are themselves the cause of several diseases, including various types of tumours, which demonstrated an altered cellular stiffness (Cross et al., 2007; Luo et al., 2016; Suresh, 2007). In this scenario, assessing the roles of cytoskeletal components in governing mechanical behaviour of the cells could provide a new tool for drug testing. Although F-actin and microtubules are assumed to be two of the most significant structural elements accounting for both mechanical stiffness and force production (Chan and Odde, 2008; Janmey, 1991; Valentine et al., 2005), the correct role of microtubules on the mechanical properties of cells is still not clear. Indeed, from literature data it appears that damaging the microtubule network left the cell stiffness unchanged (Collinsworth et al., 2002; Rotsch and Radmacher, 2000; Takai et al., 2005; Trickey et al., 2004), reduced (Nagayama and Matsumoto, 2008; Potard et al., 1997) or even increased (Stamenović et al., 2002; Wu et al., 1998; Zhou et al., 2010).

The aim of the present work is, therefore, to determine the role of both F-actin and microtubules in governing the biomechanical properties of HeLa cells, an epithelial adherent cervix cancer cell line. Exploiting the Single Cell Force Spectroscopy (SCFS) technique, we propose an extensive study to obtain important biomechanical properties both from loading-unloading curves and from stress relaxation tests. From the former, we derived (i) the cell elasticity, described by the Young's modulus, (ii) the adhesion work due to the contact of the AFM probe to the cell membrane, and (iii) the plasticity index, obtained from the hysteresis between the loading and unloading curves, which reflects the elasto-plastic behaviour of the cell. Stress relaxation data allowed a better insight of the mechanical dynamics of cells under a prolonged loading. From these data, we propose also a method to predict the mechanical spectra in terms of storage and loss moduli.

To induce morphological alterations in the cytoskeleton, HeLa cells were treated with two different well-known drugs, namely cytochalasin-D, an actin targeting toxin responsible for blocking the barbed-end of actin filaments, thereby preventing microfilament growth, or nocodazole, a microtubule-disrupting agent, which rapidly reduces the number of polymerized microtubules; a mixture 1:1 of them was also used. Although these systems have been widely studied, we believe that this work gives a better insight on the characterization of biomechanical properties of cells through AFM.

\section{Materials and methods}

\subsection{Cell culture and drug treatment}

HeLa cells (human cervix epithelioid carcinoma ATCC CCL-2) were cultured in Dulbecco's modified Eagle's medium with Glutamax (DMEM; Invitrogen, Carlsbad, CA, USA), supplemented with 10\% (v/v) foetal bovine serum (FBS), 100 units $/ \mathrm{mL}$ penicillin and $100 \mu \mathrm{g} / \mathrm{mL}$ streptomycin. After trypsinization, $1.5 \times 10^{5}$ cells per Petri dish were seeded and let adhere for $24 \mathrm{~h}$ at $37{ }^{\circ} \mathrm{C}$ and $5 \% \mathrm{CO}_{2}$, according to the standard procedure. $30 \mathrm{~min}$ before the AFM analysis, cells were treated with cytochalasin-D (Cyt-D, $5 \mu \mathrm{M}$ ), nocodazole (Noc, $10 \mu \mathrm{M}$ ), or a mixture $1: 1$ of them (Mix) and kept at $37{ }^{\circ} \mathrm{C}, 5 \% \mathrm{CO}_{2}$. Concentrations of all cytoskeletal agents were based on published research and on our own experience, to avoid cell detachment. Except for DMEM, all reagents were purchased from Sigma-Aldrich (Saint Louis, MO, USA).

\subsection{Immunofluorescence}

HeLa cells were fixed in PBS containing 4\% PFA for $20 \mathrm{~min}$ at room temperature, then aldehydes were quenched with $0.1 \mathrm{M}$ glycine in PBS for $20 \mathrm{~min}$ at room temperature. Cells were permeabilized with $0.5 \%$ Triton X-100 for $20 \mathrm{~min}$, blocked with $20 \%$ goat serum in PBS for $1 \mathrm{~h}$ at room temperature and incubated with $\alpha$-tubulin anti mouse monoclonal (T5168) antibody (Sigma-Aldrich, Saint Louis, MO, USA), at 1:4000 dilution overnight at $4{ }^{\circ} \mathrm{C}$. Cells were then washed 3 times for 10 min with PBS and $0.05 \%$ Tween 20 and finally incubated with Alexa Fluor 488-conjugated secondary antibodies (Invitrogen, Carlsbad, CA, USA) in $20 \%$ goat serum in PBS for $45-60 \mathrm{~min}$ at room temperature. Factin filaments were counterstained with Alexa Fluor 594 phalloidin (Life Technologies, Carlsbad, CA, USA) at 1:500 dilution for $45 \mathrm{~min}$ in PBS at room temperature. Additionally, nuclei were marked with TOTO-3 (Life Technologies, Carlsbad, CA, USA). For image acquisition a Nikon $\mathrm{A}^{+}$Confocal Microscope System was used. For the purpose of the study, a Plan-Apochromat $\lambda 60 \mathrm{X} / 1.40$ oil objective was used. Within each experiment, instrument settings were kept constant; all the relative acquired images are representative from at least three independent cell preparations.

\subsection{Atomic force microscopy determination of cellular biomechanics}

An AFM Solver Pro-M (NT-MDT, Moscow, RUS) was used to acquire cell morphology as well as force curves directly from Petri dishes, as previously described (Codan et al., 2014; Del Favero et al., 2012). A triangular cantilever (nominal $k=0.08 \mathrm{~N} / \mathrm{m}$ ) modified with a gold microbead (diameter around $5 \mu \mathrm{m}$, sQUBE, Bickenbach, DE) was used to precisely apply a compression force orthogonal to the nucleus, where measurements are less affected by artefacts due to the substrate stiffness. To calibrate the cantilever deflection signal, force curves versus the piezo-displacement were acquired on substrate alone. Cells can be in different stages of their life cycle, showing distinct properties that can be difficult to compare; therefore, multiple measurements from at least 20 cells were collected to obtain 'average' data for each condition of interest. Cells were selected at random as long as they had a healthy morphology (well spread) and were first scanned with AFM to define the nuclear position, corresponding to the highest portion of the cell. This was then subjected to three indentations for force-deformation tests with a rest of $20 \mathrm{~s}$ between each indentation, to allow the cell recovering. AFM tests are rate-dependent therefore, to avoid hydrodynamic forces (significant at high speed), all experiments were performed at the same speed ( $1 \mu \mathrm{m} / \mathrm{s}$ ) (Efremov et al., 2013; Mathur et al., 2001).

The total duration of the loading-unloading investigations, on a single Petri dish, was never longer than $60 \mathrm{~min}$ to avoid major variations in the physiological parameters of the cells during measurement.

\subsection{Modelling cell elasticity}

Cell elasticity was calculated from the loading curve using the Sneddon's modification of the Hertz model (Sneddon, 1965). The model assumes that the indented surface is continuous, frictionless and incompressible at small deformations: even if this is not entirely true for cells, this model is satisfactory to yield an estimation of cell elasticity and is conventionally used (Carl and Schillers, 2008). There are also other models proposed and used for assessing the cell elasticity however, since we were motivated in highlighting the differences in mechanical properties due to chemical treatments, using always the same protocol and model, the data obtained are reliable for a comparison. The Hertz-Sneddon model for spherical tips has the form (Sneddon, 1965):

$F=\frac{E}{\left(1-v^{2}\right)} \cdot\left(\frac{R^{2}+a^{2}}{2} \cdot \log \frac{R+a}{R-a}-a R\right)$

$\delta=\frac{a}{2} \cdot \log \frac{R+a}{R-a}$

Where $E$ is the Young's modulus, $F$ is the loading force, $\nu$ the Poisson ratio ( $\nu=0.5$ ), $R$ the sphere radius, $a$ is the tip contact surface radius (function of the tip penetration) and $\delta$ is the probe penetration into the 


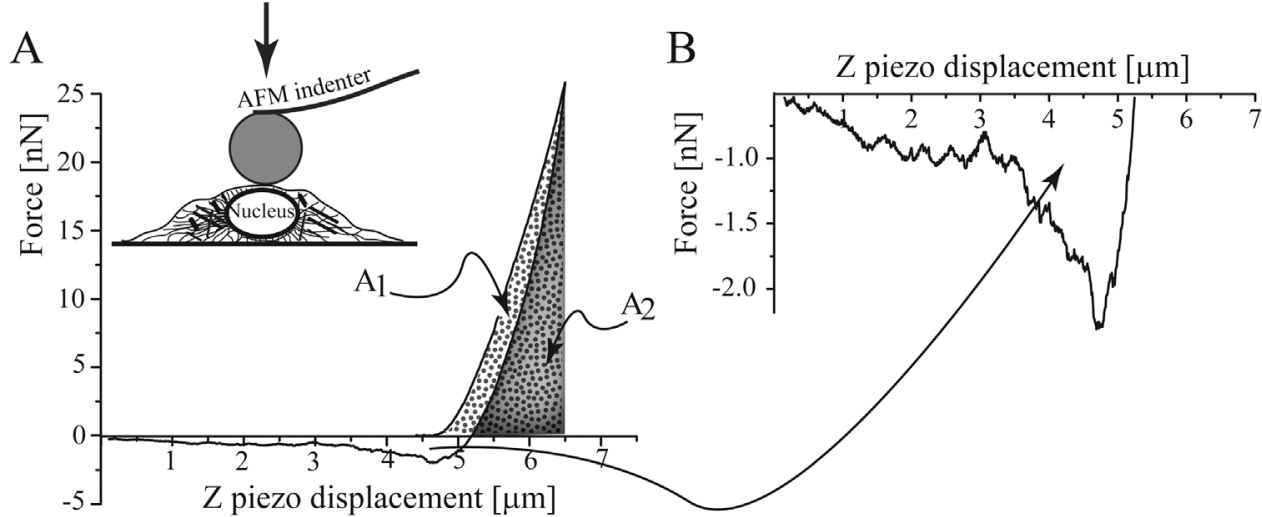

C

$\mathrm{D}$

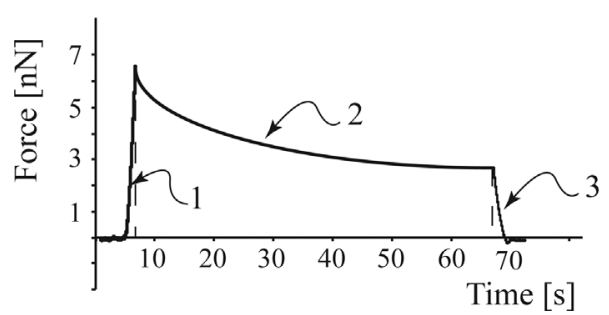

cell. The estimation of the Young's modulus was performed through the AtomicJ software (Hermanowicz et al., 2014).

\subsection{Adhesion work}

Adhesion work is considered the energy required to detach the spherical probe from the cell membrane during the unloading phase. It was evaluated with a MATLAB algorithm, integrating the area between the contact point with the cell on the loading curve and the last force interaction on the unloading one, which corresponds to the cell membrane detachment from the spherical tip (Fig. 1A and B).

\subsection{Plasticity index}

AFM force-deformation curves were also used to assess the shortterm cell viscoelastic behaviour. The indentation and retraction curves would be identical only for purely elastic cell responses, while in our cases there is a considerable difference between the two curves, as shown in Fig. 1A. This hysteresis indicates that the response is not purely elastic. To quantify the relative amount of energy that was lost due to hysteresis during the AFM experiments and then to characterize the relative elastic-plastic behaviour of the cells, we used the plasticity index $\zeta$ (Klymenko et al., 2009), a numerical parameter that can be calculated from areas under loading $\left(A_{1}\right)$ and unloading $\left(A_{2}\right)$ curves (Fig. 1A), as follows:

$\zeta=1-\left(A_{2} / A_{1}\right)$

The plasticity index varies between $\zeta=0$, if loading and unloading curves coincide and then $A_{1}=A_{2}$ (fully elastic behaviour), and $\zeta=1$, when $A_{2}=0$ (fully plastic). Intermediate values $(0<\zeta<1)$ indicate viscoelastic behaviour.

\subsection{Stress relaxation}

Stress relaxation tests were performed to assess the cell viscoelasticity under prolonged load in the so-called constant height mode (Moreno-Flores et al., 2010). Fig. 1C shows a typical time course of deflection signal during this test. In the time interval "1", the AFM tip approached the cell surface on the nuclear portion with a speed of
Fig. 1. (A) Example of a Single Cell Force Spectroscopy test carried out using the AFM on the nuclear region. $A_{1}$ and $A_{2}$ are the areas under the loading and unloading curves, respectively, and were used to evaluate the plasticity index. (B) Enlargement of the area showing where the adhesion work was calculated. The AFM sphere adheres to the cell membrane and causes an opposite deflection of the probe below the baseline during the AFM probe retraction (unloading curve). Retracting curves are characterized by a peak of force followed by a cascade of rupture events until complete detachment is achieved. (C) Example of a stress relaxation test performed on cells, showing the loading (1), relaxation (2) and unloading (3) phases. (D) Maxwell Generalized model with one pure elastic and two Maxwell elements in parallel, used to fit the stress relaxation curves.
$1 \mu \mathrm{m} / \mathrm{s}$. A script was developed to indent each cell of $30 \%$ of its initial height, which was measured from preliminary AFM scan and given as input value to the script. The movement of the piezo scanner in the $Z$ direction was corrected with the deflection of the cantilever during the cell compression. The indentation depth of $30 \%$ was chosen as a tradeoff to operate at least in quasi-linear regime from one side, and to avoid the highly-scattered data obtained at lower indentations from the other side. In the middle time interval " 2 ", the cantilever base position was kept constant for $60 \mathrm{~s}$, while the cantilever force was recorded with time. The dwell time of $60 \mathrm{~s}$ was deemed enough to reach the plateau value in the force profile and was in accordance with other studies that applied stress relaxation tests on several cell lines (Babahosseini et al., 2015; Deitch et al., 2012; Han et al., 2011; Ketene et al., 2012). In the time interval " 3 ", the AFM tip was retracted. The stress relaxation measurement was performed only one time per cell, to avoid potential artefacts due to the prolonged stress status. Each dish was investigated for a maximum duration of $60 \mathrm{~min}$.

Force data relative to the relaxation phase were expressed in terms of stress, dividing them by the contact area (calculated as projection of the spherical cap of the probe in contact with the cell after the indentation, considered constant during the relaxation phase). Stress data were then divided by deformation (indentation/initial cell height) to give a relaxation modulus $E(t)$ that can be compared with models normally used to describe linear viscoelastic behaviours. Among conventional viscoelastic models, several of them are obtained combining springs (elastic elements) and dashpots (viscous elements) in parallel and/or in series. In other studies (Koay et al., 2003; Lanzicher et al., 2015a; Wu et al., 1998, 2000) the standard linear solid (SLS) model, also called Zener's model, has been chosen to correlate the cellular viscoelastic response obtained from AFM tests, in virtue of its simplicity. It derives from the parallel arrangement of a spring and a Maxwell element, in its turn made of a spring and a dashpot combined in series. In our case the SLS model did not provide a satisfactory fitting to experimental data. Better results were obtained with a simple version of the generalized Maxwell model (GMM), i.e. the combination of a spring and two Maxwell elements in parallel (Fig. 1D). The use of three Maxwell elements (in parallel with the spring) did not improve significantly the quality of fitting and then was discarded in favour of the simpler GMM version. Accordingly, the relaxation modulus data were 
correlated with the following bi-exponential expression:

$E(t)=E_{e}+E_{1} e^{(-t / \tau 1)}+E_{2} e^{(-t / \tau 2)}$

Where $E_{\mathrm{e}}$ is the equilibrium modulus, deriving from the spring element and corresponding to asymptotic value of $E(t)$ for $t \rightarrow \infty, E_{1}$ and $E_{2}$ are the elastic moduli of the two Maxwell elements, $\tau_{1}$ and $\tau_{2}$ are the corresponding relaxation times, which govern short and long-term relaxation behaviours, respectively.

\subsection{Storage and loss moduli calculation}

In the linear viscoelastic regime, where the Boltzmann superposition is applicable, the knowledge of the relaxation modulus makes it possible to predict the rheological responses in any arbitrary deformation history, resorting to the integral version of the linear generalized viscoelastic model, where the relaxation modulus represents the fundamental kernel function (Bird et al., 1987). Consequently, also the viscoelastic responses under steady oscillatory motion can be predicted from the relaxation modulus, as long as the deformations imposed in both relaxation test and oscillatory conditions are sufficiently small, i.e. within the linear regime. In the case of the generalized Maxwell model used to fit the relaxation data, the following expressions are derived for the storage $\left(E^{\prime}\right)$ and loss moduli ( $\left.E^{\prime \prime}\right)$ as a function of the (angular) frequency $\omega$ :

$E^{\prime}(\omega)=E_{e}+E_{1} \frac{\omega^{2} \tau_{1}^{2}}{1+\omega^{2} \tau_{1}^{2}}+E_{2} \frac{\omega^{2} \tau_{2}^{2}}{1+\omega^{2} \tau_{2}^{2}}$

$E^{\prime \prime}(\omega)=E_{1} \frac{\omega \tau_{1}}{1+\omega^{2} \tau_{1}^{2}}+E_{2} \frac{\omega \tau_{2}}{1+\omega^{2} \tau_{2}^{2}}$

Such equations can serve to describe or, as in our case, to predict the mechanical spectra of the systems examined from the relaxation tests, which are assumed to be performed in quasi linear conditions.

The loss tangent, defined as the ratio between the loss modulus and the storage modulus ( $\tan \delta=E^{\prime \prime} / E^{\prime}$ ), can be used as an index of viscoelasticity, the smaller values indicating a more elastic material response.

\subsection{Statistics}

Statistical analysis was performed using GraphPad Prism software. Experimental AFM data were tested with the Shapiro-Wilk normality test $(\alpha=0.05)$ and their statistical significance, reported through the $P$-value, was probed applying the Kruskal-Wallis test; treated cells were compared to the control (not treated cells, NT) using the Dunnett's correction for multiple comparisons. A confidence interval of $95 \%$ (corresponding to $\alpha$ value of 0.05 ) was used in order to designate differences as statistically significant. Box plots are reported as median and lower/upper quartiles, while whiskers are the minimum and maximum values for each treatment.

\section{Results and discussion}

\subsection{Cell shape and morphology}

Qualitative indirect immunofluorescence analysis was performed, focusing on the different distribution/organization of both microtubules and actin microfilaments in HeLa cells not treated and after treatment with cytochalasin-D or nocodazole. Representative images obtained from confocal microscopy are illustrated in Fig. 2. The usual and most characteristic cytoskeletal organization was observed in not treated HeLa, (Fig. 2A and B), whereas treatment with Cyt-D yielded a significant fragmentation of stress fibres of F-actin, leading to the formation of actin phase dense aggregates inside the cytoplasm (Fig. $2 \mathrm{~F}$ and $\mathrm{H}$, e.g. those marked with arrows). In particular, the shape of the HeLa cells remained relatively intact, with numerous small and variable size globular structures or cytoplasmic blebs. These actin speckles coexisted with both actin microfilament bundles and diffused cortical actin network.

The use of nocodazole resulted in a microtubule disruption as confirmed by the alpha-tubulin localization (Fig. 2I and L, e.g. those marked with asterisks), with numerous small globular blebs appearing at the cell surface.

AFM imaging was also used to assess the morphology of adherent HeLa cells; from the acquired images, the cell height was defined as the distance between the highest point of the cell (corresponding to the position of the nucleus) and the substrate. Representative scan images with cross-sectional profiles are reported as Supplementary Fig. S1. The obtained height values are displayed in Fig. $3 \mathrm{~A}$ and show that Noc treated cells were taller than NT $(P<0.0001)$, Mix treated cells were lower $(P<0.0001)$, while the treatment with Cyt-D did not produce any significant difference in height compared to untreated cells.

In the widely discussed tensegrity model applied to cells (Stamenović, 2005; Stamenović et al., 2002), microtubules and F-Actin are assumed to have an antagonistic role in cytoskeletal structure, the latter providing an inward-acting contractile force that is resisted by cytoskeletal microtubules. In this configuration, disruption of the actin structure would not considerably change the cell shape, as it would be maintained by microtubules, while damaging the microtubules network would lead to cell membrane contraction and changes in cellular shape. Our results about cell morphology confirm this hypothesis: nocodazole treatment led to cell shrinkage with a contracted shape and a consequent height increase, whereas cytochalasin-D treatment did not change the cell height. Simultaneous depolymerisation of F-Actin and microtubules, instead, led to a decrease of this value, erasing the structural effect of the two cytoskeletal components. Thus, the hypothesis that the actin structures primarily carry tension seems to be rational.

\subsection{Biomechanical properties from force-deformation curves}

Elasticity data for the HeLa cells, calculated from the AFM loading curve and expressed as Young's modulus values, are reported in Fig. 3B; data for all the considered treatments displayed log-normal distributions, according to (Efremov et al., 2013). The geometric mean values of Young's modulus for Cyt-D and Mix treated cells were substantially lower $(-37 \%$ and $-64 \%$, respectively) than for NT cells, whereas stiffness almost doubled in Noc treated cells $(+129 \%)$. In accordance to other studies (Collinsworth et al., 2002; Nagayama and Matsumoto, 2008; Potard et al., 1997; Rotsch and Radmacher, 2000; Trickey et al., 2004; Wu et al., 1998), depolymerisation of F-actin, the tensile elements of the cytoskeleton, led to a reduction of the cell stiffness. On the other hand, the disruption of microtubule network gave the opposite effect: this result is similar to others reported in literature (Wu et al., 1998; Zhou et al., 2010), where an increase in cell stiffness after treatment with nocodazole was also obtained. A possible explanation is that depolymerisation of microtubule led to a change in the stress normally balanced by this structure, altering the cellular deformability and leading to cell shrinkage. This was confirmed by the observed height increase. In addition, it has been demonstrated that nocodazole can also stimulate the formation of actin fibres via a Rho signalling mechanism (Chang et al., 2008; Enomoto, 1996; Zhou et al., 2010), activating downstream Rho-associated protein kinase (ROCK) pathway. This, in turn, triggers myosin activation, ending up with an actin increased polymerization. Globally, these two effects could justify the increased cell stiffness after nocodazole treatment. In the Mix case, the simultaneous depolymerisation of F-actin and microtubules led to values of Young's modulus lower than those of nocodazole or cytochalasin-D alone, suggesting that both these cytoskeletal structures play a synergistic role in maintaining cell stiffness (Ingber et al., 1995; Wang et al., 1993).

Data regarding the adhesion work are shown in Fig. 3C. Likewise to 

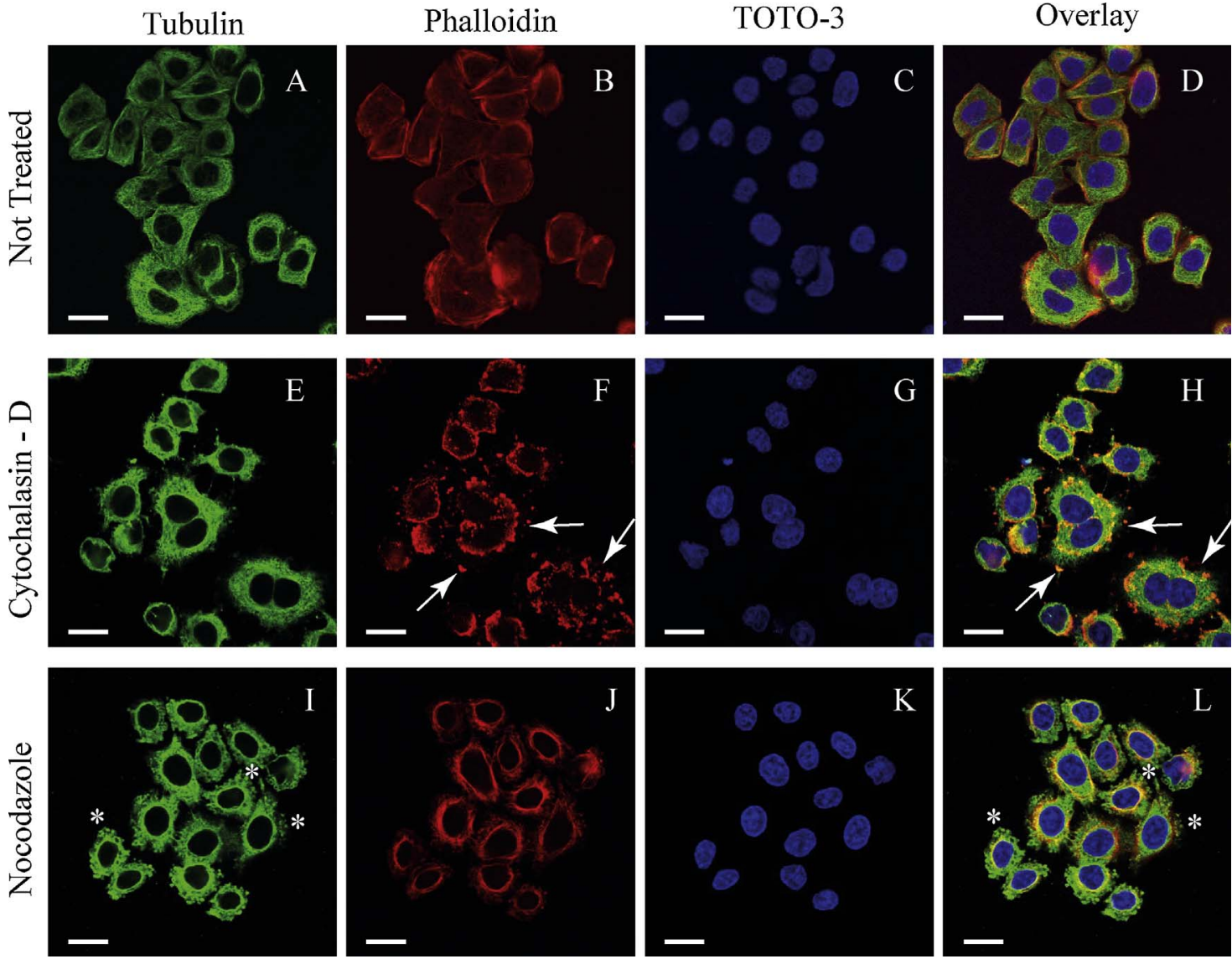

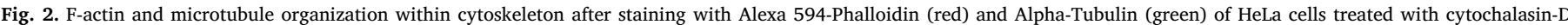

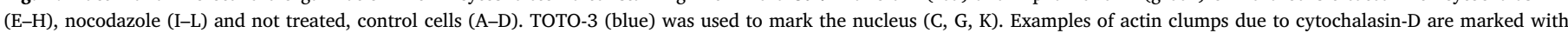

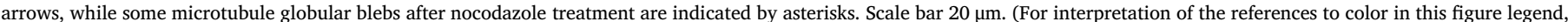
the reader is referred to the web version of this article.)

A
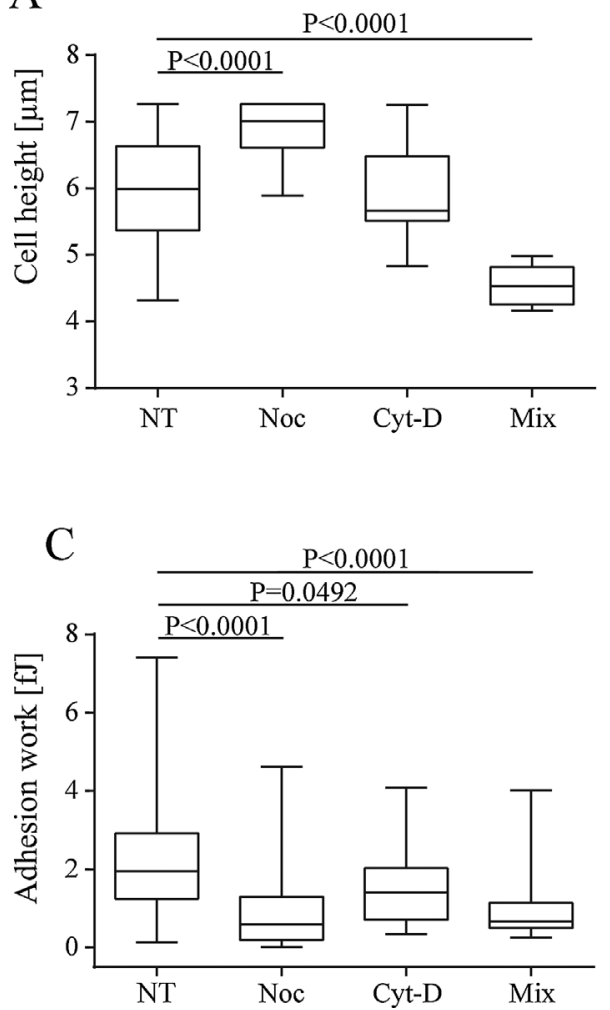

B

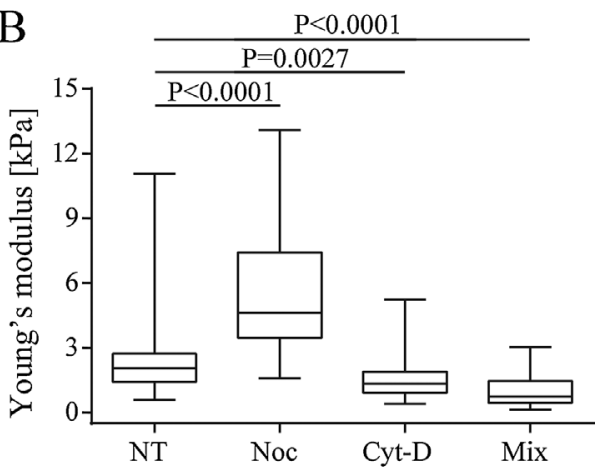

D

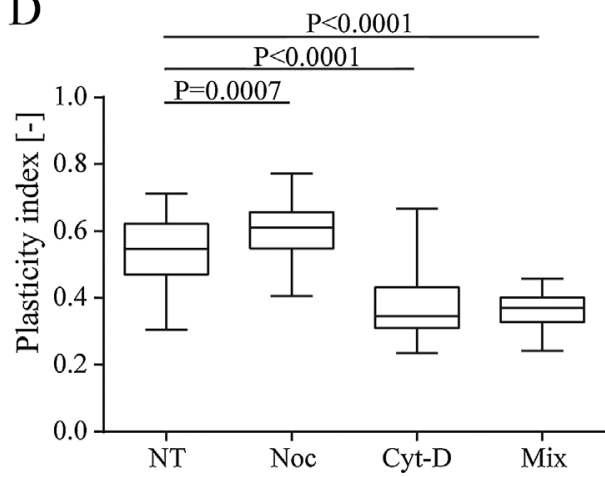

Fig. 3. Box plots relative to morphology and mechanical properties of HeLa cells after different chemical treatments: Cell height (A), Young's modulus (B), Adhesion work (C) and Plasticity Index (D). NT $=$ Not Treated,$\quad$ Noc $=$ Nocodazole,$\quad$ Cyt $\mathrm{D}=$ Cytochalasin-D, Mix = Mixture of nocodazole and cytochalasin-D. Height data were obtained from $\mathrm{n}_{\mathrm{NT}}=24$ cells, $\mathrm{n}_{\mathrm{Noc}}=27$ cells, $\mathrm{n}_{\mathrm{Cyt}-\mathrm{D}}=22$ cells and $\mathrm{n}_{\text {Mix }}=20$ cells, whereas Young's modulus, adhesion work and Plasticity Index data were relative to $\mathrm{n}_{\mathrm{NT}}=40$ cells, $\mathrm{n}_{\mathrm{Noc}}=30$ cells, $\mathrm{n}_{\text {Cyt-D }}=23$ cells and $\mathrm{n}_{\text {Mix }}=20$ cells. 
the elasticity results, they were better fitted with a log-normal distribution. The gold microbead on the cantilever was not coated with ECM components, thus the non-specific adhesion work was the resultant of van der Waals, steric and hydrophilic/hydrophobic interactions of the probe with cellular surface. In addition, the exposed thiol and amine groups of membrane proteins can interact with the gold bead. Despite its non-specific character, the use of the same protocol for all the treatments allowed to highlight the importance of F-actin and microtubule integrity in cell adhesion. Indeed, treatment with Cyt-D decreased the work of adhesion of $31 \%$, while Noc and Mix treatments further reduced these values of almost $72 \%$ and $57 \%$ compared to the control. Remarkably, values for Noc and Mix were statistically similar ( $P=0.26$, from Mann-Whitney test).

Our results confirm that an intact actin structure is essential for integrin- and cadherin-dependent adhesion, in accordance with the work of Sun et al. (Sun et al., 2005). Here, lantruculin A (another inhibitor of actin polymerization) reduced the force of tethers formed during the detachment of a silicon nitride cantilever from the cell membrane. Furthermore, previous works of our group showed reduced adhesion between gold AFM probes and cardiac cells, after cytochalasin-D treatment (Sbaizero et al., 2015) or as an effect of the D192G lamin A mutation, which was demonstrated to impair also the cytoskeletal actin organization (Lanzicher et al., 2015b). Therefore, F-actin is not only a force-bearing scaffold between sites of integrin and cadherin adhesion, but it provides a direct physical basis for adhesive network interactions

The strong reduction of the observed values due to nocodazole, greater than what obtained with cytochalasin-D, suggests that also microtubules network plays an important role in the adhesion machinery. Indeed, microtubules develop from the centrosome, grow towards the cell substrate and push the integrins to adhere to the extracellular matrix. They are also anchored and regulated by cadherins, and their terminations are stabilized by a linkage to the cell membrane through integrin adhesions (Hotta et al., 2010), providing another potential cytoskeleton-based mechanism for adhesive networking. The effect of Mix treatment on the adhesion work, being comparable to the one of nocodazole, further stresses the role of microtubule in the cellular adhesive properties.

The plasticity index (Fig. 3D) reflected the altered viscoelastic behaviour of the treated cells compared to the NT control and emphasized the effect of the different cytoskeletal components under fast loadingunloading perturbations. The plasticity index for Noc treated cells was higher than that of the NT cells $(+14 \%)$ while data collected for both Cyt-D and Mix treated cells were statistically similar $(P=0.47$, from Mann-Whitney test) and smaller than NT cells ( $-31 \%$ in both cases). The increased plasticity index after nocodazole treatment indicates that microtubule depolymerisation, in addition to an increased cell stiffness, led to a more viscous system, restricting the mobility of small molecules in the cytoskeleton to flow away from the area where stress is applied. On the other hand, depolymerisation of F-actin, both on cytochalasin-D and on Mix treated cells, seems to fluidize the cytoskeleton, leading to a less viscous system.

\subsection{Stress relaxation and derived mechanical spectra}

Converting the force relaxation profiles in terms of modulus versus time data and fitting them to a bi-exponential model, parameters such as the initial and the equilibrium elastic moduli $\left(E_{\text {tot }}\right.$ and $E_{e}$ ) and the two characteristic relaxation times were derived. In particular, $E_{\text {tot }}$ is the sum of the three elastic moduli $E_{e}, E_{1}$ and $E_{2}$ of the Generalized Maxwell model used to fit the relaxation curves and represents the elastic modulus at the end of the indentation phase, while $E_{e}$ is the elastic modulus at the end of relaxation $(t \rightarrow \infty)$.

The obtained parameters are reported in Fig. 4A and B (elastic moduli $E_{\text {tot }}$ and $E_{e}$ ) and Fig. 4C and D (decay times $\tau_{1}$ and $\tau_{2}$ ). Actin microfilaments are expected to play an important role on the viscoelastic behaviour of cells. Indeed, both $E_{\text {tot }}$ and $E_{e}$ were significantly reduced $(-62 \%$ and $-83 \%$, respectively) with Cyt-D treatment; conversely, Noc treatment caused their increase $\left(E_{\text {tot }}:+121 \%, E_{e}\right.$ : $+193 \%$ ) while, apparently, no significant effect was produced by Mix treatment on these moduli compared to the control. Depolymerisation of F-actin by cytochalasin-D reduced also the decay times $\left(\tau_{1}=-27 \%\right.$, $\tau_{2}=-36 \%$ ), while the differences produced by the other treatments were not statistically significant in comparison with the control.

Due to their cross-linked cytoskeletal network, cells exhibit viscoelastic behaviour, having either the ability to store energy or to dissipate it through viscous effects. Thus, it is possible to separate the viscous and elastic components as well as to evaluate their relative weight in energy terms when cells are subjected to stresses or strains of small amplitude with different rates of application, i.e. at different frequencies. Indeed, the mechanical energy stored in the cell during loading and totally recovered during unloading is proportional to the in-phase component $\left(E^{\prime}\right)$, while the energy irreversibly converted into heat is measured by the out-of-phase component ( $E$ "). The relative weight of the two terms is given by $\tan \delta$ and usually varies with the characteristic time of the sinusoidal input $(t=1 / \omega)$.

The calculated profiles of storage modulus $E^{\prime}(\omega)$ and of loss modulus $E "(\omega)$ are shown in Fig. 5A, while in Fig. 5B the corresponding frequency dependence of loss tangent $(\tan \delta)$ is reported. The obtained mechanical spectra are qualitatively similar, since they were obtained from the same simple version of the Generalized Maxwell model (GMM), i.e. the parallel combination of a purely elastic and two Maxwell elements. The traces of the storage modulus $E$ ' are similar and confined within two asymptotic values, the equilibrium modulus $E_{\mathrm{e}}$ (for $\omega \rightarrow 0$ ) and the high frequency limit $E_{\text {tot }}=E_{\mathrm{e}}+E_{1}+E_{2}($ for $\omega \rightarrow \infty)$, which correspond to the asymptotic and initial values of the relaxation modulus $E(t)$, respectively.

At low $\omega, E^{\prime}$ is close to the corresponding $E_{\mathrm{e}}$ value and increases in the following order: Cyt-D $<$ Mix $<$ NT $<$ Noc; at high frequencies, the order is: Cyt-D $<$ NT $<$ Mix $<$ Noc, so that in the intermediate $\omega$ interval the curves of Mix and NT cross over each other.

All $E$ " traces have two symmetric asymptotic branches $(E " \rightarrow 0)$ at low and high frequencies (for $\omega \rightarrow 0$ and $\omega \rightarrow \infty$ ), respectively, and two maximum values and/or curvatures at frequencies equal to the two reciprocal relaxation times, $1 / \tau_{1}$ and $1 / \tau_{2}$. The differences between systems are more marked at low $\omega$, where the increasing order of $E$ " (Cyt-D $<$ NT $<$ Mix $<$ Noc) replicates that obtained from relaxation tests for $E_{2}$ (the elastic modulus of the Maxwell elements with the higher relaxation time, $\tau_{2}$ ). At high frequencies, differences become less important or negligible: the profiles of Cyt-D and NT systems overlap, while Mix values slightly exceed all the others.

The tangent delta curves highlight similarities and differences in the viscoelastic character of the cells under examination (Fig. 5B). Noc treatment strengthens the elastic character, whereas opposite effects can be observed for Cyt-D and Mix cells. In the last case, the combined action of cytochalasin-D and nocodazole seems to produce a negative synergic effect in the low frequency region, where the elastic character of the cells decreases even more than for Cyt-D treatment.

The storage and the loss moduli directly reveal fundamental features of our systems. It is well established that cross-linked networks have a storage modulus much larger than loss modulus and are stiffer than the entangled solutions (Lin et al., 2007). As shown by our data, E' always exceeds $E$ " value; furthermore, a distinct minimum and two maxima in the loss modulus are observed for all cell lines: this behaviour is similar to what has been reported also for more regular systems, as linear cross-linked (Satmarel et al., 2003) and dendrimeric copolymers (Satmarel et al., 2004), and can be seen as connection of springs with two different elasticity. The highest peak in the $E$ " profile corresponds to the energy loss by the less stiff springs due to the friction with the viscous medium. The peak values and their ratio depend on the cross-link density, whereas their positions do not: indeed, the amount of energy dissipation increases with the cross-link density and can thus be 


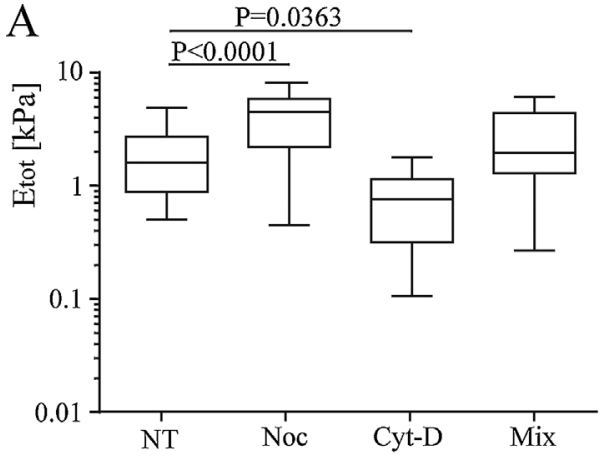

$\mathrm{C}$

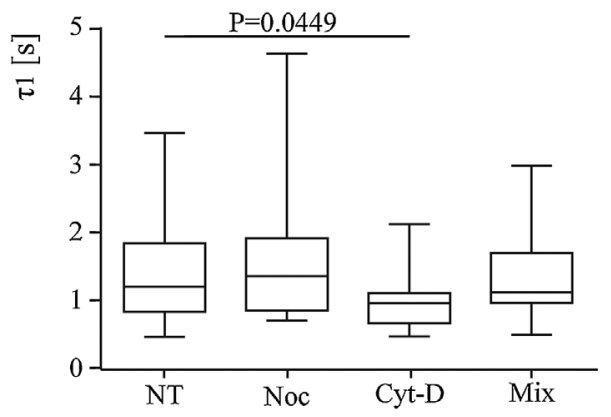

B

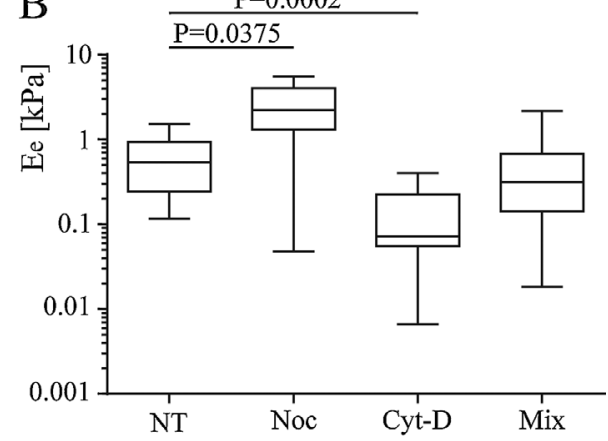

$\mathrm{D}$

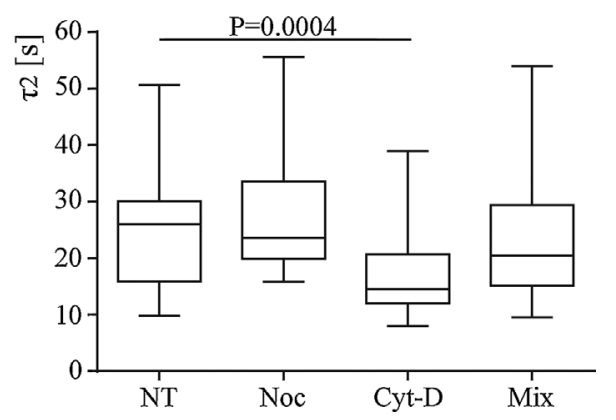

Fig. 4. Box plots relative to the elastic moduli $E_{\text {tot }}$ (A) and $E_{e}$ (B) and the two decay times $\tau_{1}$ (C) and $\tau_{2}$ (D) obtained from the stress relaxation tests on HeLa cells after different chemical treatments. NT $=$ Not Treated, Noc $=$ Nocodazole, Cyt-D $=$ CytochalasinD, Mix $=$ Mixture of nocodazole and cytochalasin-D. Data were obtained from $\mathrm{n}_{\mathrm{NT}}=22$ cells, $\mathrm{n}_{\mathrm{Noc}}=30$ cells, $\mathrm{n}_{\mathrm{Cyt}-\mathrm{D}}=27$ cells and $\mathrm{n}_{\mathrm{Mix}}=27$ cells. identified as a direct result of the competition between local stress release triggered by cross-link unbinding and the friction of single filaments. Furthermore, the relative height of the two peaks also sheds light into the system composition, since the height of the peaks is related to the number of cross-links of one kind involved in the energy dissipation around a given frequency.

The values of $E_{\text {tot }}, E_{e}$ from stress relaxation tests and the derived mechanical spectra for Noc are in accordance with both the Young's modulus (increased stiffness) and the plasticity index (increased viscosity) values obtained from force-deformation curves, in comparison with NT cells. The $E$ ' and $E$ " profiles for nocodazole treatment reflect the increased density of chain entanglements and filamentous networks with strong permanent cross-links and could justify the hypothesis of a more crowded system, due to both the failure of microtubules (which normally balance the inward F-actin stress effect) and the already discussed increased F-actin polymerization from the activation of the Rho pathway.

Conversely, data relative to cytochalasin-D treatment showed a dramatic decrease compared to the control, according to what was obtained from the loading-unloading curves. With the actin cytoskeleton mostly disassembled, the more fluid-like cytoplasm controls the viscoelastic behaviour, as already outlined by the reduced plasticity index. In addition, the very low values of both $E^{\prime}$ and $E$ " (the loss modulus is comparable to the control only at high frequencies) are symptomatic of a weak physical network, susceptible to changes by thermal energy at physiological temperatures, where filaments are essentially entangled rather than cross-linked. It has been already shown that upon administration of Cyt-D, the actin filaments close to the cell membrane are readily rearranged into short rods-like filaments (Foissner and Wasteneys, 2007). This causes the formation of variably oriented but locally aligned short actin filaments, the density of which differs along the cell surface. Such a local structure could resemble that of a nematic viscoelastic fluid, formed by rod-like elements preferentially aligned along a common direction, but with no positional order. Interestingly, the $E$ " profile after Cyt-D treatment is the only one with the second peak higher than the first one, confirming that in this case the short-time relaxation, associated to smaller and more mobile structures, dominates the viscous component of the cell.

The relaxation behaviour for the Mix treated cells seems counterintuitive if compared to what obtained from the force-deformation
A

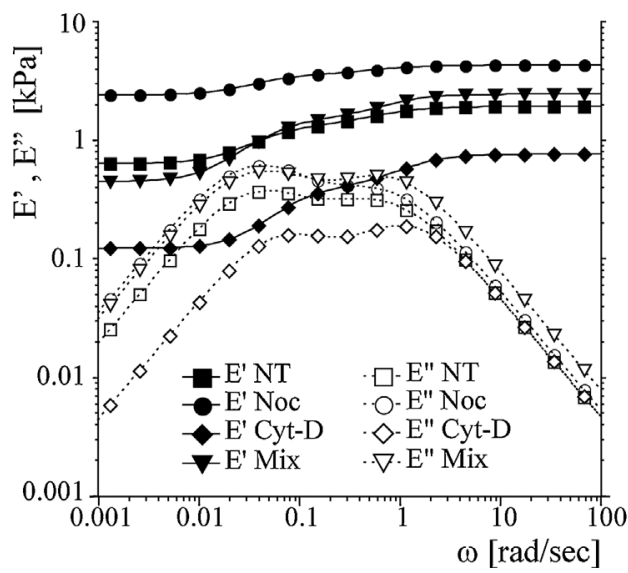

$\mathrm{B}$

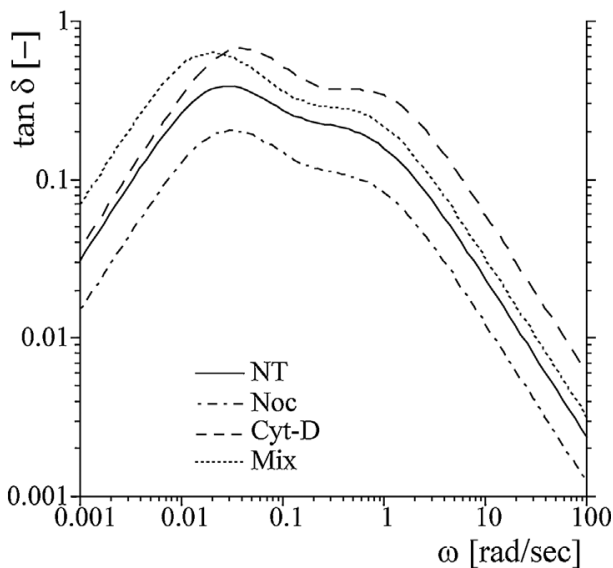

Fig. 5. Storage moduli (E', continuous lines), loss moduli (E”, dashed lines) (A) and loss tangent (B) profiles versus the angular frequency $\omega$, as calculated from stress relaxation data of Fig. 4. NT $=$ Not Treated, Noc $=$ Nocodazole, Cyt-D $=$ CytochalasinD, Mix $=$ Mixture of nocodazole and cytochalasin-D 
curves, where a lower Young's modulus and a reduced plasticity index were found. $E_{\text {tot }}, E_{e}$ and decay times resulted statistically similar to the values of NT cells, resulting in profiles of $E$ ' and $E$ " very close to the one of the control. Based on the cell height data, we speculated that the $30 \%$ indentation depth led to a higher stress exerted directly on the nucleus, which is not balanced by the depolymerized F-actin and microtubule cytoskeletal structure.

\section{Conclusions}

Our study showed that force-deformation curves and stress relaxation test with AFM can be used to extensively characterize the biomechanical properties of cells and to derive their mechanical behaviour under oscillatory regime. With these tools, the mechanical modifications induced by different drugs can be determined and compared.

In the case of HeLa cells, AFM data showed that disruption of F-actin microfilaments decreased the cell stiffness (Young's modulus), the adhesion work and both the plasticity index and the viscoelasticity behaviour, shifting toward a more fluid-like cell behaviour. Disassembling the microtubule network resulted instead to increase the cell stiffness and the plasticity index, with a more solid-like behaviour, whereas the adhesion work was reduced.

Although the drugs used in this work have been widely studied for their effects on biomechanics, sometimes with contrasting results, the findings here obtained for nocodazole treatment could suggest another mechanism for drug actions addressed to avoid metastatic spreading. It has been consistently shown that, in cancer progression, the formation of metastases poses the biggest threat, being metastases significantly softer than normal cells (Luo et al., 2016; Suresh, 2007). However, there is scant information about cell biomechanics due to the fact that mechanical properties of cells are governed by a multitude of passive and active elements that are far from being completely understood. Undeniably, cancer drugs that affect microtubule formation have been used for many years and their function mainly consists in inhibiting cells mitosis; nonetheless, our results suggest an additional possibility to drastically change the biomechanical properties of metastases, making them stiffer and therefore less prone to diffuse.

\section{Author contributions}

The work presented here was carried out in collaboration between all authors. OS, LP, DB developed the original idea and defined the research theme. LP and DB co-designed methods and experiments. MC, LP, DB and VM carried out the laboratory experiments, analyzed the data, discussed analyses and interpreted the results. OS drafted the manuscript; DB and LP carried out a critical revision of the manuscript for important intellectual content and discussed presentation. RL took care of the model to derive storage and loss moduli from relaxation data. All authors have contributed to, seen and approved the manuscript.

\section{Conflicts of interest}

None.

\section{Acknowlegments}

Authors are thankful to Prof. Massimo Borelli and Ilaria Pecorari from University of Trieste for their help in performing statistical analysis of experimental data. Financial support from "Foreman-Casali" Foundation, Trieste (Italy) to VM.

\section{Appendix A. Supplementary data}

Supplementary data associated with this article can be found, in the online version, at http://dx.doi.org/10.1016/j.micron.2017.09.002.

\section{References}

Babahosseini, H., Carmichael, B., Strobl, J.S., Mahmoodi, S.N., Agah, M., 2015. Subcellular force microscopy in single normal and cancer cells. Biochem. Biophys. Res. Commun. 463, 587-592. http://dx.doi.org/10.1016/j.bbrc.2015.05.100.

Beloussov, L.V., Saveliev, S.V., Naumidi, I.I., Novoselov, V.V., 1994. Mechanical stresses in embryonic tissues: patterns, morphogenetic role, and involvement in regulatory feedback. Int. Rev. Cytol. 1-34. http://dx.doi.org/10.1016/S0074-7696(08)61535-1.

Binnig, G., Quate, C.F., Gerber, C., 1986. Atomic force microscope. Phys. Rev. Lett. 56, 930-933. http://dx.doi.org/10.1103/PhysRevLett.56.930.

Bird, R.B., Armstrong, R.C., Hassager, O., 1987. Dynamics of Polymeric Liquids Vol 1: Fluid Mechanics, 2nd edition. Wiley-Interscience, New York.

Carl, P., Schillers, H., 2008. Elasticity measurement of living cells with an atomic force microscope: data acquisition and processing. Pflugers Arch. Eur. J. Physiol. 457, 551-559. http://dx.doi.org/10.1007/s00424-008-0524-3.

Chan, C.E., Odde, D.J., 2008. Traction dynamics of filopodia on compliant substrates. Science 322, 1687-1691. http://dx.doi.org/10.1126/science.1163595.

Chang, Y.-C., Nalbant, P., Birkenfeld, J., Chang, Z.-F., Bokoch, G.M., 2008. 3GEF-H1 couples nocodazole-induced microtubule disassembly to cell contractility via RhoA. Mol. Biol. Cell 19, 2147-2153. http://dx.doi.org/10.1091/mbc.E07-12-1269.

Codan, B., Del Favero, G., Martinelli, V., Long, C.S., Mestroni, L., Sbaizero, O., 2014. Exploring the elasticity and adhesion behavior of cardiac fibroblasts by atomic force microscopy indentation. Mater. Sci. Eng. C 40, 427-434. http://dx.doi.org/10.1016/ j.msec. 2014.04.003.

Collinsworth, A.M., Zhang, S., Kraus, W.E., Truskey, G.A., 2002. Apparent elastic modulus and hysteresis of skeletal muscle cells throughout differentiation. AJP Cell Physiol. 283, C1219-C1227. http://dx.doi.org/10.1152/ajpcell.00502.2001.

Cross, S.E., Jin, Y.-S., Rao, J., Gimzewski, J.K., 2007. Nanomechanical analysis of cells from cancer patients. Nat. Nanotechnol. 2, 780-783. http://dx.doi.org/10.1038/ nnano.2007.388.

Deitch, S., Gao, B.Z., Dean, D., 2012. Effect of matrix on cardiomyocyte viscoelastic properties in 2D culture. Mol. Cell. Biomech. 9, 227-249.

Del Favero, G., Florio, C., Codan, B., Sosa, S., Poli, M., Sbaizero, O., Molgó, J., Tubaro, A., Lorenzon, P., 2012. The stretch-activated channel blocker Gd $3+$ reduces palytoxin toxicity in primary cultures of skeletal muscle cells. Chem. Res. Toxicol. 25, 1912-1920. http://dx.doi.org/10.1021/tx300203x.

Duncan, R.L., Turner, C.H., 1995. Mechanotransduction and the functional response of bone to mechanical strain. Calcif. Tissue Int. 57, 344-358. http://dx.doi.org/10. 1007/BF00302070.

Efremov, Y.M., Dokrunova, A.A., Bagrov, D.V., Kudryashova, K.S., Sokolova, O.S., Shaitan, K.V., 2013. The effects of confluency on cell mechanical properties. J. Biomech. 46, 1081-1087. http://dx.doi.org/10.1016/j.jbiomech.2013.01.022.

Enomoto, T., 1996. Microtubule disruption induces the formation of actin stress fibers and focal adhesions in cultured cells: possible involvement of the rho signal cascade. Cell Struct. Funct. 21, 317-326. http://dx.doi.org/10.1247/csf.21.317.

Foissner, I., Wasteneys, G.O., 2007. Wide-ranging effects of eight cytochalasins and latrunculin A and B on intracellular motility and actin filament reorganization in characean internodal cells. Plant Cell Physiol. 48, 585-597. http://dx.doi.org/10. 1093/pcp/pcm030.

Gorfien, S.F., Winston, F.K., Thibault, L.E., Macarak, E.J., 1989. Effects of biaxial deformation on pulmonary artery endothelial cells. J. Cell. Physiol. 139, 492-500. http://dx.doi.org/10.1002/jcp.1041390307.

Han, L., Frank, E.H., Greene, J.J., Lee, H.Y., Hung, H.H.K., Grodzinsky, A.J., Ortiz, C., 2011. Time-dependent nanomechanics of cartilage. Biophys. J. 100, 1846-1854. http://dx.doi.org/10.1016/j.bpj.2011.02.031.

Hermanowicz, P., Sarna, M., Burda, K., Gabryś, H., 2014. AtomicJ: an open source software for analysis of force curves. Rev. Sci. Instrum. 85, 63703. http://dx.doi.org/10. 1063/1.4881683.

Hotta, A., Kawakatsu, T., Nakatani, T., Sato, T., Matsui, C., Sukezane, T., Akagi, T., Hamaji, T., Grigoriev, I., Akhmanova, A., Takai, Y., Mimori-Kiyosue, Y., 2010. Laminin-based cell adhesion anchors microtubule plus ends to the epithelial cell basal cortex through LL5 $\alpha / \beta$. J. Cell Biol. 189, 901-917. http://dx.doi.org/10.1083/jcb. 200910095.

Huang, H., Kamm, R.D., Lee, R.T., 2004. Cell mechanics and mechanotransduction: pathways, probes, and physiology. Am. J. Physiol. Cell Physiol. 287, C1-C11. http:// dx.doi.org/10.1152/ajpcell.00559.2003.

Ingber, D.E., Prusty, D., Sun, Z., Betensky, H., Wang, N., 1995. Cell shape, cytoskeletal mechanics, and cell cycle control in angiogenesis. J. Biomech. 28, 1471-1484. http:// dx.doi.org/10.1016/0021-9290(95)00095-X.

Isenberg, B.C., Tranquillo, R.T., 2003. Long-term cyclic distention enhances the mechanical properties of collagen-based media-equivalents. Ann. Biomed. Eng. 31, 937-949. http://dx.doi.org/10.1114/1.1590662.

Janmey, P.A., 1991. Mechanical properties of cytoskeletal polymers. Curr. Opin. Cell Biol. 3, 4-11. http://dx.doi.org/10.1016/0955-0674(91)90159-V.

Ketene, A.N., Schmelz, E.M., Roberts, P.C., Agah, M., 2012. The effects of cancer progression on the viscoelasticity of ovarian cell cytoskeleton structures. Nanomed. Nanotechnol. Biol. Med. 8, 93-102. http://dx.doi.org/10.1016/j.nano.2011.05.012.

Kim, B.-S., Nikolovski, J., Bonadio, J., Mooney, D.J., 1999. Cyclic mechanical strain regulates the development of engineered smooth muscle tissue. Nat. Biotechnol. 17, 979-983. http://dx.doi.org/10,1038/13671.

Klymenko, O., Wiltowska-Zuber, J., Lekka, M., Kwiatek, W.M., 2009. Energy dissipation in the AFM elasticity measurements. Acta Phys. Pol. A 115, 548-551. http://dx.doi. org/10.12693/APhysPolA.115.548.

Koay, E.J., Shieh, A.C., Athanasiou, K.A., 2003. Creep indentation of single cells. J. Biomech. Eng. 125, 334-341. http://dx.doi.org/10.1115/1.1572517. 
Kulik, T.J., Alvarado, S.P., 1993. Effect of stretch on growth and collagen synthesis in cultured rat and lamb pulmonary arterial smooth muscle cells. J. Cell. Physiol. 157, 615-624. http://dx.doi.org/10.1002/jcp.1041570322.

Lammi, M.J., 2004. Current perspectives on cartilage and chondrocyte mechanobiology. Biorheology 41, 593-596.

Lanzicher, T., Martinelli, V., Long, C.S., Del Favero, G., Puzzi, L., Borelli, M., Mestroni, L., Taylor, M.R.G., Sbaizero, O., 2015a. AFM single-cell force spectroscopy links altered nuclear and cytoskeletal mechanics to defective cell adhesion in cardiac myocytes with a nuclear lamin mutation. Nucleus 6, 394-407. http://dx.doi.org/10.1080/ 19491034.2015.1084453.

Lanzicher, T., Martinelli, V., Puzzi, L., Del Favero, G., Codan, B., Long, C.S., Mestroni, L., Taylor, M.R.G., Sbaizero, O., 2015b. The cardiomyopathy lamin A/C D192G mutation disrupts whole-cell biomechanics in cardiomyocytes as measured by atomic force microscopy loading-unloading curve analysis. Sci. Rep. 5, 13388. http://dx.doi.org/ 10.1038/srep13388.

Li, C., Xu, Q., 2007. Mechanical stress-initiated signal transduction in vascular smooth muscle cells in vitro and in vivo. Cell. Signal. 19, 881-891. http://dx.doi.org/10. 1016/j.cellsig.2007.01.004.

Lin, Y.C., Koenderink, G.H., MacKintosh, F.C., Weitz, D.A., 2007. Viscoelastic properties of microtubule networks. Macromolecules 40, 7714-7720. http://dx.doi.org/10. 1021/ma0708621.

Luo, Q., Kuang, D., Zhang, B., Song, G., 2016. Cell stiffness determined by atomic force microscopy and its correlation with cell motility. Biochim. Biophys. Acta - Gen. Subj. 1860, 1953-1960. http://dx.doi.org/10.1016/j.bbagen.2016.06.010.

Mathur, A.B., Collinsworth, A.M., Reichert, W.M., Kraus, W.E., Truskey, G.A., 2001. Endothelial, cardiac muscle and skeletal muscle exhibit different viscous and elastic properties as determined by atomic force microscopy. J. Biomech. 34, 1545-1553. http://dx.doi.org/10.1016/S0021-9290(01)00149-X.

Moreno-Flores, S., Benitez, R., Vivanco dM, M., Toca-Herrera, J.L., 2010. Stress relaxation microscopy: imaging local stress in cells. J. Biomech. 43, 349-354. http://dx.doi.org/ 10.1016/j.jbiomech.2009.07.037.

Nagayama, K., Matsumoto, T., 2008. Contribution of actin filaments and microtubules to quasi-in situ tensile properties and internal force balance of cultured smooth muscle cells on a substrate. Am. J. Physiol. Cell Physiol. 295, C1569-C1578. http://dx.doi. org/10.1152/ajpcell.00098.2008.

Potard, U.S., Butler, J.P., Wang, N., 1997. Cytoskeletal mechanics in confluent epithelial cells probed through integrins and E-cadherins. Am. J. Physiol. 272, C1654-63.

Rotsch, C., Radmacher, M., 2000. Drug-induced changes of cytoskeletal structure and mechanics in fibroblasts: an atomic force microscopy study. Biophys. J. 78, 520-535. http://dx.doi.org/10.1016/S0006-3495(00)76614-8.

Sarraf, C.E., Harris, A.B., McCulloch, A.D., Eastwood, M., 2002. Tissue engineering of biological cardiovascular system surrogates. Heart Lung Circ. 11, 142-150. http://dx. doi.org/10.1046/j.1444-2892.2002.00150.x.

Satmarel, C., Gurtovenko, A.A., Blumen, A., 2003. Viscoelastic relaxation of cross-linked, alternating copolymers in the free-draining limit. Macromolecules 36, 486-494. http://dx.doi.org/10.1021/ma0214409.

Satmarel, C., Gurtovenko, A.A., Blumen, A., 2004. Relaxation of copolymeric dendrimers built from alternating monomers. Macromol. Theory Simul. 13, 487-496. http://dx. doi.org $/ 10.1002 /$ mats. 200400020 .
Sbaizero, O., DelFavero, G., Martinelli, V., Long, C.S., Mestroni, L., 2015. Analysis of long and short-range contribution to adhesion work in cardiac fibroblasts: an atomic force microscopy study. Mater. Sci. Eng. C 49, 217-224. http://dx.doi.org/10.1016/j.msec. 2014.12.083.

Seliktar, D., Nerem, R.M., Galis, Z.S., 2001. The role of matrix metalloproteinase-2 in the remodeling of cell-seeded vascular constructs subjected to cyclic strain. Ann. Biomed. Eng. 29, 923-934. http://dx.doi.org/10.1114/1.1415522.

Simpson, D.G., Carver, W., Borg, T.K., Terracio, L., 1994. Role of mechanical stimulation in the establishment and maintenance of muscle cell differentiation. Int. Rev. Cytol. 69-94. http://dx.doi.org/10.1016/S0074-7696(08)61537-5.

Sneddon, I.N., 1965. The relation between load and penetration in the axisymmetric boussinesq problem for a punch of arbitrary profile. Int. J. Eng. Sci. 3, 47-57. http:// dx.doi.org/10.1016/0020-7225(65)90019-4.

Stamenović, D., Liang, Z., Chen, J., Wang, N., 2002. Effect of the cytoskeletal prestress on the mechanical impedance of cultured airway smooth muscle cells. J. Appl. Physiol. 92, 1443-1450. http://dx.doi.org/10.1152/japplphysiol.00782.2001.

Stamenović, D., 2005. Effects of cytoskeletal prestress on cell rheological behavior. Acta Biomater. 1, 255-262. http://dx. doi.org/10.1016/j. actbio.2005.01.004.

Sun, M., Graham, J.S., Hegedüs, B., Marga, F., Zhang, Y., Forgacs, G., Grandbois, M., 2005. Multiple membrane tethers probed by atomic force microscopy. Biophys. J. 89, 4320-4329. http://dx.doi.org/10.1529/biophysj.104.058180.

Suresh, S., 2007. Biomechanics and biophysics of cancer cells. Acta Mater. 55, 3989-4014. http://dx.doi.org/10.1016/j.actamat.2007.04.022.

Takai, E., Costa, K.D., Shaheen, A., Hung, C.T., Guo, X.E., 2005. Osteoblast elastic modulus measured by atomic force microscopy is substrate dependent. Ann. Biomed. Eng. 33, 963-971. http://dx.doi.org/10.1007/s10439-005-3555-3.

Trickey, W.R., Vail, T.P., Guilak, F., 2004. The role of the cytoskeleton in the viscoelastic properties of human articular chondrocytes. J. Orthop. Res. 22, 131-139. http://dx. doi.org/10.1016/S0736-0266(03)00150-5.

Valentine, M.T., Perlman, Z.E., Mitchison, T.J., Weitz, D.A., 2005. Mechanical propertie of Xenopus egg cytoplasmic extracts. Biophys. J. 88, 680-689. http://dx.doi.org/10. 1529/biophysj.104.048025.

Van Essen, D.C., 1997. A tension-based theory of morphogenesis and compact wiring in the central nervous system. Nature. http://dx.doi.org/10.1038/385313a0.

Vande Geest, J.P., Di Martino, E.S., Vorp, D.A., 2004. An analysis of the complete strain field within FlexercellTM membranes. J. Biomech. 37, 1923-1928. http://dx.doi.org/ 10.1016/j.jbiomech.2004.02.022.

Wang, N., Butler, J., Ingber, D., 1993. Mechanotransduction across the cell surface and through the cytoskeleton. Science 260 (80), 1124-1127. http://dx.doi.org/10.1126/ science.7684161.

Wu, H.W., Kuhn, T., Moy, V.T., 1998. Mechanical properties of L929 cells measured by atomic force microscopy: effects of anticytoskeletal drugs and membrane crosslinking. Scanning 20, 389-397. http://dx.doi.org/10.1002/sca.1998.4950200504.

Wu, Z.Z., Zhang, G., Long, M., Wang, H.B., Song, G.B., Cai, S.X., 2000. Comparison of the viscoelastic properties of normal hepatocytes and hepatocellular carcinoma cells under cytoskeletal perturbation. Biorheology 37, 279-290.

Zhou, J., Kim, H.Y., Wang, J.H.-C., Davidson, L.A., 2010. Macroscopic stiffening of embryonic tissues via microtubules, RhoGEF and the assembly of contractile bundles of actomyosin. Development 137, 2785-2794. http://dx.doi.org/10.1242/dev.045997. 